\title{
Novel mutations in the glucocerebrosidase gene of Indian patients with Gaucher disease
}

Chitra Ankleshwaria, Mehul Mistri, Ashish Bavdekar, Mamta Muranjan, Usha Dave, Parag Tamhankar, Varun Khanna, Eresha Jasinge, Sheela Nampoothiri, Suresh Edayankara Kadangot, Frenny Sheth, Sarita Gupta and Jayesh Sheth

Journal of Human Genetics (2015) 60, 285; doi:10.1038/jhg.2015.27

Correction to: Journal of Human Genetics (2014) 59, 223-228. doi:10.1038/jhg.2014.5; published online 13 February 2014.

The authors of the above paper noticed errors in Table 2 on publication that are shown below:

1. Change patient \#22's genotype from L444P (c.1448T >C)/L444P (c.1448T > C) to L444P (c.1448T > C)/R496C (c.1603C > T).

2. Change patient \#31's genotype from L444P (c.1448T $>$ C)/L444P (c.1448T > C) to L444P (c.1448T > C)/R329C (c.1093G >A).
3. Change patient \#33's genotype from I466S (c.1397T > G)/? to I427S (c.1397T > G)/?.

4. Change patient \#29's genotype from G289A (c.866 G>C)/G289A (c.866 G > C) to G250A (c.866 G>C)/G250A (c.866 G>C).

These corrections do not alter the results and their interpretation as discussed in the paper.

The authors would like to apologize for these mistakes. 